\title{
Three-Dimensional Extensions of Resolvability of Two Point Targets in MIMO Radar Using a Hypothesis Test Formulation
}

\author{
Ning Zhang, Jun Tang, Shuang Wan, and Wei Zhu \\ Department of Electronic Engineering, Tsinghua University, Beijing 100084, China \\ Correspondence should be addressed to Ning Zhang; n-zhang11@mails.tsinghua.edu.cn
}

Received 13 December 2013; Revised 29 April 2014; Accepted 5 May 2014; Published 25 May 2014

Academic Editor: Se-Yun Kim

Copyright (c) 2014 Ning Zhang et al. This is an open access article distributed under the Creative Commons Attribution License, which permits unrestricted use, distribution, and reproduction in any medium, provided the original work is properly cited.

\begin{abstract}
Multiple-input multiple-output (MIMO) radar has been extensively studied in recent years, due to its outstanding performance in various aspects. Resolution and estimation of the targets' parameters are central capabilities of a MIMO radar system. In this paper, the resolution performance of MIMO radar in terms of the three-dimensional statistical resolution limit (3D SRL) is investigated, where three-dimension refers to direction of arrival (DOA), direction of departure (DOD), and Doppler frequency of a target. The scenario of two closely spaced targets corrupted by broadband noise with/without clutter sources is considered and the closedform expression of the resolution threshold, which is the minimum signal-to-noise ratio (SNR) required for a given resolution performance, is derived using a generalized likelihood ratio test (GLRT) formulation. Finally, both theoretical and numerical analyses are provided for some insights regarding the resolution limit.
\end{abstract}

\section{Introduction}

Multiple-input multiple-output (MIMO) radar has been extensively studied in recent years, due to its advantages such as improved parameter identifiability, direct applicability of adaptive algorithms, and enhanced target detection and estimation performance [1-3]. The performance improvements of MIMO radar primarily benefit from spatial and waveform diversity. More precisely, either the array configuration or the transmitted waveforms can be chosen rather freely in contrast with its phased-array counterpart [4-7].

Resolution and estimation of the targets' parameters are central capabilities of MIMO radar, where the former is the theme of this paper. To be specific, the essential resolution performance of MIMO radar described by the statistical resolution limit (SRL) $[8,9]$ is our concern. SRL is an important measure to characterize the resolution ability which has been rising in several applications recently [10-14]. In general, there exist three essential manners to characterize the SRL $[8,9]$.

(1) The first approach is relevant to spectral estimation algorithms based on the eigen-decomposition of the data covariance matrix, including MUSIC, ESPRIT, or their variants $[15,16]$. The Cox criterion $[8]$ and the Sharman and Durrani criterion [17] have been developed to define the resolvability in this context.

(2) The second approach is based on estimation accuracy criteria that are independent of specific algorithms, where the Cramer-Rao bound (CRB) and some other minimal bounds tighter [14] are employed to describe the SRL. Smith criterion [13] and its multidimensional generalization [18] are widely used to evaluate the resolution performance in this framework.

(3) The last one, which is the strategy adopted in this work, is based on the hypothesis test theory $[19,20]$. The original idea of this method is to determine from the observation the presence of one or two signals/targets. In this spirit, SRL is defined as the minimum separation that allows a correct resolvability for a given probability of false alarm and probability of detection. Usually the closed-form expression of SRL can be obtained for one-dimensional parameter. As for the multidimensional case, the main concern turns to calculating the resolution threshold [21], that is, the minimum signal-to-noise ratio (SNR) 
or signal-to-clutter-plus-noise ratio (SCNR) required for a given resolution performance.

Several works have been done on SRL of MIMO radar in existing literatures. For instance, in [22], the asymptotic SRL was derived using the Smith criterion. The scenario discussed was a colocated MIMO radar system working in an environment with stationary clutters and the sole parameter of interest was the target's direction of arrival (DOA). In [9], MIMO radar with widely separated transmit and receive arrays was considered and the resolution threshold for two targets imbedded in moving clutter interferences was derived. Particularly, the interested parameter therein became twodimensional, that is, DOA and direction of departure (DOD). In addition, the Doppler frequencies of the two targets to be resolved were constrained to be equal and known as a prior. The authors claimed that the cases of unequal or unknown Doppler are intractable. However, this rigid assumption cannot be satisfied in most practical cases and thus has become the main obstacle for the wide usage of this scheme. In this work, we extend the formulation in [9] to the three-dimensional (3D) case. As we will see later, the aforementioned Doppler constraint is unnecessary and the difference between the Doppler frequencies of the two targets can further be exploited for resolution. Obviously this extension can greatly broaden the applicable occasions as well as providing a thorough performance evaluation for general cases.

The paper is organized as follows. Both the signal model and the resolution strategy we adopted are provided in Section 2. The closed-form expressions of the resolution thresholds in the cases of known/unknown noise variance with/without clutter interferences are derived in Section 3. In Section 4, numerical analyses of the resolution thresholds are given to provide some insights into the theoretical results. Finally, Section 5 concludes the paper.

Notation. Throughout this paper, matrices are denoted by bold capital letters and vectors are denoted by bold lowercase letters. $P_{\mathbf{X}}$ and $P_{\mathbf{X}}^{\perp}$ denote the orthogonal projector onto the subspace spanned by the columns of the matrix $\mathbf{X}$ and its orthogonal complement subspace, respectively. Superscripts $(\cdot)^{T}$ and $(\cdot)^{H}$ denote transpose and conjugate transpose correspondingly. vec $(\cdot)$ means the vectorization operation. $\mathbf{I}_{K}$ denotes the identity matrix of size $K \times K .\|\cdot\|^{2}$ represents the Euclidean norm of a vector. $\otimes$ and $\odot$ denote the Kronecker and Hadamard products, respectively. $Q_{d}^{-1}(\cdot)$ denotes the right tail of the probability density function of $d$-distribution. Finally, complex Gaussian distribution is denoted by $\mathscr{C} \mathscr{N}(\cdot, \cdot)$.

\section{Problem Formulation}

2.1. Original Observation Model and Its Linear Approximation. Consider a MIMO radar system consisting of $K$ transmitters and $L$ receivers where both the transmit and the receive arrays are colocated linear arrays and they can be widely spaced (e.g., see Figure 1). In this work, we assume the transmitted waveforms to be mutually orthogonal signals with normalized energy; that is, $\mathbf{S S}^{H}=\mathbf{I}_{K}$, where

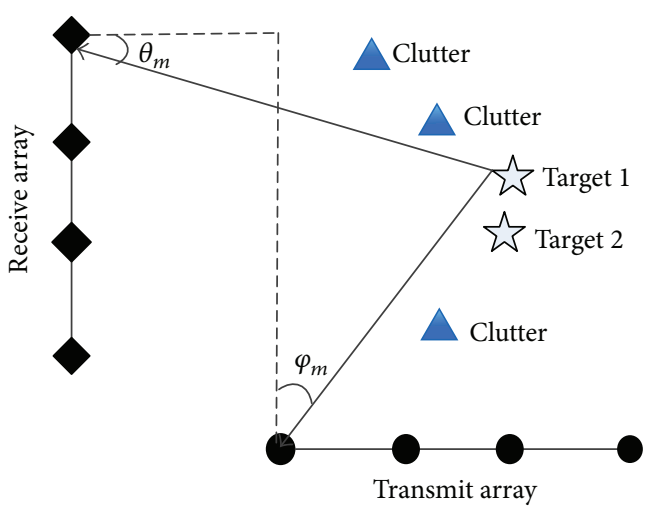

Figure 1: Two closely spaced targets imbedded in three clutter sources.

$\mathbf{S}=\left[\mathbf{s}_{0}, \mathbf{s}_{1}, \ldots, \mathbf{s}_{K-1}\right]^{T}$. To facilitate the description of the echoes from moving targets, the coherent pulse train model is adopted where $N$ pulses constitute a coherent processing interval (CPI) and the pulse repetition interval (PRI) is T [23].

Suppose that $M$ targets are present in a certain range cell; then the output of the receive array in the $n$th pulse period of a CPI can be written as (omit the relative delay)

$$
\begin{array}{r}
\mathbf{X}_{n}=\sum_{m=1}^{M} \alpha_{m} e^{j w_{m}^{(D)} n} \mathbf{a}_{R}\left(w_{m}^{(R)}\right) \mathbf{a}_{T}\left(w_{m}^{(T)}\right)^{T} \mathbf{S}+\mathbf{W}_{n}, \\
n=0,1, \ldots, N-1,
\end{array}
$$

where $\alpha_{m}$ denotes the complex scattering coefficient of target $m$ while the $i$ th elements of $\mathbf{a}_{R}\left(w_{m}^{(R)}\right)$ and $\mathbf{a}_{T}\left(w_{m}^{(T)}\right)$ are given by $e^{j w_{m}^{(R)} d_{i}^{(R)}}$ and $e^{j w_{m}^{(T)} d_{i}^{(T)}}$, in which $d_{i}^{(R)}$ and $d_{i}^{(T)}$ represent the distance between the $i$ th antenna and the reference antenna in the receive and transmit arrays, respectively. The 3D parameters of target $m$ satisfy $\left[w_{m}^{(D)}, w_{m}^{(T)}, w_{m}^{(R)}\right]=$ $\left[4 \pi v_{m} T / \lambda, 2 \pi \sin \left(\varphi_{m}\right) / \lambda, 2 \pi \sin \left(\theta_{m}\right) / \lambda\right]$, where $\lambda$ is the wavelength and $v_{m}, \varphi_{m}$, and $\theta_{m}$ denote the radial velocity, DOD, and DOA of it. In addition, the elements in the noise matrix $\mathbf{W}_{n}$ are assumed to be independent identically distributed (i.i.d.) complex Gaussian random variables with zero mean and variance $\sigma^{2}$.

A sufficient statistic matrix for the targets' parameters can be obtained via matched filtering as [3]

$$
\begin{aligned}
\mathbf{Y}_{n}=\mathbf{X}_{n} \mathbf{S}^{H}= & \sum_{m=1}^{M} \boldsymbol{\alpha}_{m} e^{j w_{m}^{(D)} n} \mathbf{a}_{R}\left(w_{m}^{(R)}\right) \mathbf{a}_{T}\left(w_{m}^{(T)}\right)^{T} \\
& +\mathbf{Z}_{n}, \quad n=0,1, \ldots, N-1
\end{aligned}
$$

where $\mathbf{Z}_{n}=\mathbf{W}_{n} \mathbf{S}^{H}$. Applying the vectorization operation to (2) yields a concise expression

$$
\mathbf{y}=\mathbf{x}+\mathbf{z}
$$


where

$$
\begin{aligned}
& \mathbf{y}=\left[\operatorname{vec}\left(\mathbf{Y}_{0}\right)^{T}, \ldots, \operatorname{vec}\left(\mathbf{Y}_{N-1}\right)^{T}\right]^{T}, \\
& \mathbf{x}=\sum_{m=1}^{M} \alpha_{m} \mathbf{g}_{m}, \\
& \mathbf{z}=\left[\operatorname{vec}\left(\mathbf{Z}_{0}\right)^{T}, \ldots, \operatorname{vec}\left(\mathbf{Z}_{N-1}\right)^{T}\right]^{T} \triangleq\left[\mathbf{z}_{0}^{T}, \ldots, \mathbf{z}_{N-1}^{T}\right]^{T}, \\
& \mathbf{g}_{m}=\mathbf{a}_{D}\left(w_{m}^{(D)}\right) \otimes \mathbf{a}_{T}\left(w_{m}^{(T)}\right) \otimes \mathbf{a}_{R}\left(w_{m}^{(R)}\right), \\
& {\left[\mathbf{a}_{D}\left(w_{m}^{(D)}\right)\right]_{i}=e^{j w_{m}^{(D)} i}, \quad i=0,1, \ldots, N-1,} \\
& {\left[\mathbf{a}_{T}\left(w_{m}^{(T)}\right)\right]_{i}=e^{j w_{m}^{(T)} d_{i}^{(T)}}, \quad i=0,1, \ldots, K-1,} \\
& {\left[\mathbf{a}_{R}\left(w_{m}^{(R)}\right)\right]_{i}=e^{j w_{m}^{(R)} d_{i}^{(R)}}, \quad i=0,1, \ldots, L-1 .}
\end{aligned}
$$

As for the statistical property of the vector $\mathbf{z}$, we have [9]

$$
\begin{aligned}
E\left(\mathbf{z}_{n} \mathbf{z}_{n^{\prime}}^{H}\right) & =\left(\mathbf{S}^{*} \otimes \mathbf{I}_{L}\right) E\left(\operatorname{vec}\left(\mathbf{W}_{n}\right) \operatorname{vec}\left(\mathbf{W}_{n^{\prime}}\right)^{H}\right)\left(\mathbf{S}^{T} \otimes \mathbf{I}_{L}\right) \\
& =\sigma^{2} \mathbf{I}_{K L} \delta\left(n-n^{\prime}\right)
\end{aligned}
$$

which translate into $E\left(\mathbf{z z}^{H}\right)=\sigma^{2} \mathbf{I}_{K L N}$; that is,

$$
\mathbf{y} \sim \mathscr{C} \mathcal{N}\left(\mathbf{x}, \sigma^{2} \mathbf{I}_{K L N}\right)
$$

Without loss of generality, we assume that the first two targets are of interest and the remaining $M-2$ targets are clutter sources. Obviously in the scenario considered here, the two targets to be resolved are in the vicinity of each other in each dimension (otherwise, some regular techniques, e.g., beamforming or moving target detection (MTD), can be directly employed to accomplish the resolution). This precondition promotes us to use an approximation version of the original model $[9,24]$. We will elaborate the details in the sequel.

Firstly, several auxiliary variables are introduced as

$$
\begin{aligned}
& w_{c}^{(D)}=\frac{w_{2}^{(D)}+w_{1}^{(D)}}{2}, \quad w_{c}^{(T)}=\frac{w_{2}^{(T)}+w_{1}^{(T)}}{2}, \\
& w_{c}^{(R)}=\frac{w_{2}^{(R)}+w_{1}^{(R)}}{2}, \quad \delta_{D}=w_{2}^{(D)}-w_{1}^{(D)}, \\
& \delta_{T}=w_{2}^{(T)}-w_{1}^{(T)}, \quad \delta_{R}=w_{2}^{(R)}-w_{1}^{(R)},
\end{aligned}
$$

where the triple $\left(\delta_{D}, \delta_{T}, \delta_{R}\right)$ constitute the 3D SRL. Subsequently, we launch the first order Taylor expansion on the steering vectors $\mathbf{a}_{D}\left(w_{m}^{(D)}\right), \mathbf{a}_{T}\left(w_{m}^{(T)}\right)$, and $\mathbf{a}_{R}\left(w_{m}^{(R)}\right)$ around $\left(\delta_{D}, \delta_{T}, \delta_{R}\right)=(0,0,0)$; thus we have

$$
\begin{aligned}
& \mathbf{a}_{D}\left(w_{1}^{(D)}\right)=\mathbf{a}_{D}\left(w_{c}^{(D)}\right)-\frac{j}{2} \delta_{D} \mathbf{a}_{D}\left(w_{c}^{(D)}\right) \odot \mathbf{d}_{D}, \\
& \mathbf{a}_{D}\left(w_{2}^{(D)}\right)=\mathbf{a}_{D}\left(w_{c}^{(D)}\right)+\frac{j}{2} \delta_{D} \mathbf{a}_{D}\left(w_{c}^{(D)}\right) \odot \mathbf{d}_{D}, \\
& \mathbf{a}_{T}\left(w_{1}^{(T)}\right)=\mathbf{a}_{T}\left(w_{c}^{(T)}\right)-\frac{j}{2} \delta_{T} \mathbf{a}_{T}\left(w_{c}^{(T)}\right) \odot \mathbf{d}_{T}, \\
& \mathbf{a}_{T}\left(w_{2}^{(T)}\right)=\mathbf{a}_{T}\left(w_{c}^{(T)}\right)+\frac{j}{2} \delta_{T} \mathbf{a}_{T}\left(w_{c}^{(T)}\right) \odot \mathbf{d}_{T}, \\
& \mathbf{a}_{R}\left(w_{1}^{(R)}\right)=\mathbf{a}_{R}\left(w_{c}^{(R)}\right)-\frac{j}{2} \delta_{R} \mathbf{a}_{R}\left(w_{c}^{(R)}\right) \odot \mathbf{d}_{R}, \\
& \mathbf{a}_{R}\left(w_{2}^{(R)}\right)=\mathbf{a}_{R}\left(w_{c}^{(R)}\right)+\frac{j}{2} \delta_{R} \mathbf{a}_{R}\left(w_{c}^{(R)}\right) \odot \mathbf{d}_{R},
\end{aligned}
$$

where

$$
\begin{aligned}
& \mathbf{d}_{D}=[0,1, \ldots, N-1]^{T}, \\
& \mathbf{d}_{T}=\left[d_{0}^{(T)}, d_{1}^{(T)}, \ldots, d_{K-1}^{(T)}\right]^{T}, \\
& \mathbf{d}_{R}=\left[d_{0}^{(R)}, d_{1}^{(R)}, \ldots, d_{L-1}^{(R)}\right]^{T} .
\end{aligned}
$$

Substituting (8) into (3) gives the following linear model:

$$
\mathrm{y}=\mathrm{G} \zeta+\mathrm{D} \alpha+\mathrm{z},
$$

where

$$
\begin{gathered}
\mathbf{G}=\left[\mathbf{v}_{2}, \mathbf{v}_{3}, \ldots, \mathbf{v}_{8}\right], \\
\mathbf{D}=\left[\mathbf{v}_{1}, \mathbf{g}_{3}, \ldots, \mathbf{g}_{M}\right], \\
\boldsymbol{\zeta}=\frac{j}{2}\left[\delta_{R}\left(\alpha_{2}-\alpha_{1}\right), \delta_{T}\left(\alpha_{2}-\alpha_{1}\right),\right. \\
\frac{j}{2} \delta_{R} \delta_{T}\left(\alpha_{2}+\alpha_{1}\right), \delta_{D}\left(\alpha_{2}-\alpha_{1}\right), \\
\frac{j}{2} \delta_{D} \delta_{R}\left(\alpha_{2}+\alpha_{1}\right), \frac{j}{2} \delta_{D} \delta_{T}\left(\alpha_{2}+\alpha_{1}\right), \\
\left.\frac{1}{4} \delta_{D} \delta_{T} \delta_{R}\left(\alpha_{1}-\alpha_{2}\right)\right]^{T}, \\
\boldsymbol{\alpha}=\left[\alpha_{1}+\alpha_{2}, \alpha_{3}, \ldots, \alpha_{M}\right]^{T}
\end{gathered}
$$


in which

$$
\begin{aligned}
\mathbf{v}_{1}= & \mathbf{a}_{D}\left(w_{c}^{(D)}\right) \otimes \mathbf{a}_{T}\left(w_{c}^{(T)}\right) \otimes \mathbf{a}_{R}\left(w_{c}^{(R)}\right), \\
\mathbf{v}_{2}= & \mathbf{a}_{D}\left(w_{c}^{(D)}\right) \otimes \mathbf{a}_{T}\left(w_{c}^{(T)}\right) \otimes\left(\mathbf{a}_{R}\left(w_{c}^{(R)}\right) \odot \mathbf{d}_{R}\right), \\
\mathbf{v}_{3}= & \mathbf{a}_{D}\left(w_{c}^{(D)}\right) \otimes\left(\mathbf{a}_{T}\left(w_{c}^{(T)}\right) \odot \mathbf{d}_{T}\right) \otimes \mathbf{a}_{R}\left(w_{c}^{(R)}\right) \\
\mathbf{v}_{4}= & \mathbf{a}_{D}\left(w_{c}^{(D)}\right) \otimes\left(\mathbf{a}_{T}\left(w_{c}^{(T)}\right) \odot \mathbf{d}_{T}\right) \otimes\left(\mathbf{a}_{R}\left(w_{c}^{(R)}\right) \odot \mathbf{d}_{R}\right) \\
\mathbf{v}_{5}= & \left(\mathbf{a}_{D}\left(w_{c}^{(D)}\right) \odot \mathbf{d}_{D}\right) \otimes \mathbf{a}_{T}\left(w_{c}^{(T)}\right) \otimes \mathbf{a}_{R}\left(w_{c}^{(R)}\right), \\
\mathbf{v}_{6}= & \left(\mathbf{a}_{D}\left(w_{c}^{(D)}\right) \odot \mathbf{d}_{D}\right) \otimes \mathbf{a}_{T}\left(w_{c}^{(T)}\right) \otimes\left(\mathbf{a}_{R}\left(w_{c}^{(R)}\right) \odot \mathbf{d}_{R}\right), \\
\mathbf{v}_{7}= & \left(\mathbf{a}_{D}\left(w_{c}^{(D)}\right) \odot \mathbf{d}_{D}\right) \otimes\left(\mathbf{a}_{T}\left(w_{c}^{(T)}\right) \odot \mathbf{d}_{T}\right) \otimes \mathbf{a}_{R}\left(w_{c}^{(R)}\right), \\
\mathbf{v}_{8}= & \left(\mathbf{a}_{D}\left(w_{c}^{(D)}\right) \odot \mathbf{d}_{D}\right) \otimes\left(\mathbf{a}_{T}\left(w_{c}^{(T)}\right) \odot \mathbf{d}_{T}\right) \\
& \otimes\left(\mathbf{a}_{R}\left(w_{c}^{(R)}\right) \odot \mathbf{d}_{R}\right) ;
\end{aligned}
$$

we will mainly use this simplified model in the rest of this paper.

2.2. Hypothesis Test Formulation of the 3D Resolution Problem. As a generalization of the work in [9], we formulate the problem of resolving two close targets in terms of the 3D SRL as the following binary hypothesis test:

$$
\begin{aligned}
& H_{0}:\left(\delta_{D}, \delta_{T}, \delta_{R}\right)=(0,0,0), \\
& H_{1}:\left(\delta_{D}, \delta_{T}, \delta_{R}\right) \neq(0,0,0) .
\end{aligned}
$$

The clear implication of (13) is that only if we can discriminate the two targets from any dimension of the parameter space, they are resolvable. Plugging (13) into the linear observation model in (10) gives an equivalent form of the hypothesis test

$$
\begin{gathered}
H_{0}: \mathbf{y}=\mathbf{D} \boldsymbol{\alpha}+\mathbf{z} \sim \mathscr{C} \mathcal{N}\left(\mathbf{D} \boldsymbol{\alpha}, \sigma^{2} \mathbf{I}_{K L N}\right), \\
H_{1}: \mathbf{y}=\mathbf{G} \zeta+\mathbf{D} \boldsymbol{\alpha}+\mathbf{z} \sim \mathscr{C} \mathscr{N}\left(\mathbf{G} \zeta+\mathbf{D} \boldsymbol{\alpha}, \sigma^{2} \mathbf{I}_{K L N}\right),
\end{gathered}
$$

where the 3D SRL is included in the vector $\boldsymbol{\zeta}$ and $\boldsymbol{\alpha}$ consists only of nuisance scattering coefficients.

Note that unknown parameters exist in both hypotheses; thus it is impossible to design an optimal detector in the Neyman-Pearson sense [19]. Alternatively, the widely used invariant detector [19] generalized likelihood ratio test (GLRT) is employed to investigate the resolution performance. For the convenience of the subsequent derivation, we further assume that the matrices $\mathbf{G}$ and $\mathbf{D}$ are known or previously estimated. One should note that without these priors, the solution of the GLRT is intractable and consequently is beyond the scope of this paper.

\section{Derivation of the Resolution Threshold}

The closed-form expressions of the resolution thresholds in terms of the $3 \mathrm{D}$ SRL are derived in the sequel.
The discussions are divided into three parts, where both the cases of known/unknown noise variance are considered, and finally the performance of the so-called clairvoyant detector [9] is studied as a performance evaluation indicator.

3.1. Case of Known Noise Variance. We first study the case that the noise variance $\sigma^{2}$ is known. Under such circumstance, the maximum likelihood estimate (MLE) of the unknowns in (14) is given by [25]

$$
\begin{aligned}
& \widehat{\boldsymbol{\alpha}}_{H_{0}}=\left(\mathbf{D}^{H} \mathbf{D}\right)^{-1} \mathbf{D}^{H} \mathbf{y}, \\
& \widehat{\boldsymbol{\alpha}}_{H_{1}}=\left(\mathbf{D}^{H} P_{\mathbf{G}}^{\perp} \mathbf{D}\right)^{-1} \mathbf{D}^{H} P_{\mathbf{G}}^{\perp} \mathbf{y}, \\
& \widehat{\boldsymbol{\zeta}}=\left(\mathbf{G}^{H} P_{\mathbf{D}}^{\perp} \mathbf{G}\right)^{-1} \mathbf{G}^{H} P_{\mathbf{D}}^{\perp} \mathbf{y} .
\end{aligned}
$$

Consequently, the log-GLRT statistic can be defined as

$$
\begin{aligned}
& T_{K}(\mathbf{y}) \\
& =2 \ln \frac{p\left(\mathbf{y} ; \widehat{\boldsymbol{\alpha}}_{H_{1}}, \widehat{\boldsymbol{\zeta}}, H_{1}\right)}{p\left(\mathbf{y} ; \widehat{\boldsymbol{\alpha}}_{H_{0}}, H_{0}\right)}=\frac{2}{\sigma^{2}}\left(\left\|\widehat{\mathbf{z}}_{H_{0}}\right\|^{2}-\left\|\widehat{\mathbf{z}}_{H_{1}}\right\|^{2}\right) \\
& =\frac{2}{\sigma^{2}} \mathbf{y}^{H}\left(P_{\mathbf{D}}^{\perp}-P_{[\mathbf{G D}]}^{\perp}\right) \mathbf{y}=\frac{2}{\sigma^{2}} \mathbf{y}^{H} P_{P_{\mathbf{D}}^{\perp} \mathbf{G}} \mathbf{y},
\end{aligned}
$$

where $\widehat{\mathbf{z}}_{H_{0}}=\mathbf{y}-\mathbf{D} \widehat{\boldsymbol{\alpha}}_{H_{0}}=P_{\mathbf{D}}^{\perp} \mathbf{y}$ and $\widehat{\mathbf{z}}_{H_{1}}=\mathbf{y}-\mathbf{G} \widehat{\boldsymbol{\zeta}}-\mathbf{D} \widehat{\boldsymbol{\alpha}}_{H_{1}}=$ $P_{[\mathbf{G D}]}^{\perp} \mathbf{y}$. Let $P_{P_{\mathbf{D}}^{\perp} \mathbf{G}}=\mathbf{U U}^{H}$ be an orthogonal decomposition of the projector and define $\widetilde{\mathbf{y}}=\mathbf{U}^{H} \mathbf{y}$; obviously $T_{K}(\mathbf{y})=$ $2\|\tilde{\mathbf{y}}\|^{2} / \sigma^{2}$ holds and an immediate consequence is

$$
T_{K}(\mathbf{y}) \sim\left\{\begin{array}{l}
H_{0}: \chi_{2 r}^{2} \\
H_{1}: \chi_{2 r}^{2}\left(\lambda_{K}\right),
\end{array}\right.
$$

where the degree of freedom (DOF) and the noncentrality parameter of the chi-square distribution are given by

$$
\begin{gathered}
r=\operatorname{rank}\left(P_{P_{\mathbf{D}}^{\perp} \mathbf{G}}\right)=\operatorname{rank}(\mathbf{G})=7, \\
\lambda_{K}=\frac{2 \zeta^{H} \mathbf{G}^{H} P_{P_{\mathbf{D}}^{\perp} \mathbf{G}} \mathbf{G} \zeta}{\sigma^{2}}=\frac{2 \zeta^{H} \mathbf{G}^{H} P_{\mathbf{D}}^{\perp} \mathbf{G} \zeta}{\sigma^{2}} .
\end{gathered}
$$

By investigating the expressions of $r$ and $\lambda_{K}$ in (18), some inferences regarding the influence of the clutters on the resolution performance ensue.

(1) The intensities of the clutters, that is, $\alpha_{3}, \ldots, \alpha_{M}$, are not related with the resolution performance. It is straightforward to conclude that the definition of SCNR is meaningless in this work; therefore we only focus on SNR hereafter.

(2) Recall that $\mathbf{D}=\left[\mathbf{v}_{1}, \mathbf{g}_{3}, \ldots, \mathbf{g}_{M}\right] \triangleq\left[\mathbf{v}_{1}, \mathbf{D}^{\prime}\right]$; hence $P_{\mathbf{D}}^{\perp} \mathbf{G}=\left(\mathbf{I}_{K L N}-P_{\left[\mathbf{v}_{1}, \mathbf{D}^{\prime}\right]}\right) \mathbf{G}=\left(\mathbf{I}_{K L N}-P_{\mathbf{v}_{1}}-P_{P_{\mathbf{v}_{1}}^{\perp} \mathbf{D}^{\prime}}\right) \mathbf{G}$ holds. Clearly the influences of the clutters are fully embodied in the projector $P_{P_{\mathbf{v}_{1}}^{\perp} \mathbf{D}^{\prime}}$, which promotes us to further define the column space of $P_{\mathbf{v}_{1}}^{\perp} \mathbf{D}^{\prime}$ as a remaining clutter space. $\forall m \in[3, \ldots, M], \mathbf{g}_{m}=\mathbf{v}_{1}$ 
holds when the condition $\left[w_{m}^{(D)}, w_{m}^{(T)}, w_{m}^{(R)}\right]=\left[w_{c}^{(D)}\right.$, $\left.w_{c}^{(T)}, w_{c}^{(R)}\right]$ meets, which translates into the fact that clutter $m$ has no effect on the remaining clutter space, or to say, it appears to be transparent. Consider an extreme case that the column space of $\mathbf{D}^{\prime}$ locates in the space spanned by $\mathbf{v}_{1}$; then an immediate consequence is that the remaining clutter space is a null space; hence all the clutters will appear to be transparent under such circumstance.

The quantitative performance of a hypothesis test is usually evaluated by the couple composed of the false alarm probability $P_{F}$ and the detection probability $P_{D}$. Specific to our problem, they represent the probability of sucessfully resolving two close targets and incorrectly split a single target into two individual ones, respectively. Having conveyed the basic ideas and intuition, now we are left of establishing the expression of the aforementioned resolution threshold. In view of the distribution given in (17), the noncentrality parameter $\lambda_{K}$ can be numerically computed as the solution of

$$
Q_{\chi_{2 r}^{2}}^{-1}\left(P_{F}\right)=Q_{\chi_{2 r}^{2}\left(\lambda_{K}\right)}^{-1}\left(P_{D}\right)
$$

for a fixed $\left(P_{D}, P_{F}\right)$. Consequently, the resolution threshold can be defined as

$$
\mathrm{SNR}_{K} \triangleq \frac{\left|\alpha_{1}\right|^{2}+\left|\alpha_{2}\right|^{2}}{\sigma^{2}}=\frac{\lambda_{K}\left(\left|\alpha_{1}\right|^{2}+\left|\alpha_{2}\right|^{2}\right)}{2 \zeta^{H} \mathbf{G}^{H} P_{\mathbf{D}}^{\perp} \mathbf{G} \zeta} .
$$

For a special case that the two targets are in a clutter free environment, $\mathrm{SNR}_{K}$ can be expressed in a more compact way. To be specific, using the property of the oblique projector [25], the term $\mathbf{G}^{H} P_{\mathbf{D}}^{\perp} \mathbf{G}$ in the denominator of (20) can be further written as

$$
\begin{aligned}
\mathbf{G}^{H} P_{\mathbf{D}}^{\perp} \mathbf{G} & =\mathbf{G}^{H}\left(P_{\mathbf{v}_{1}}^{\perp}-P_{\left[\mathbf{v}_{1} \mathbf{G}\right]}^{\perp}\right) \mathbf{G} \\
& =\mathbf{G}^{H}\left(P_{\left[\mathbf{v}_{1} \mathbf{G}\right]}-P_{\mathbf{v}_{1}}\right) \mathbf{G} \\
& =\mathbf{G}^{H}\left(E_{\mathbf{v}_{1} \mathbf{G}}+E_{\mathbf{G}_{1}}-P_{\mathbf{v}_{1}}\right) \mathbf{G} \\
& =\mathbf{G}^{H} \mathbf{G}-\mathbf{G}^{H} P_{\mathbf{v}_{1}} \mathbf{G} \\
& =\Phi-\frac{N}{K L} \kappa \kappa^{H},
\end{aligned}
$$

where

$$
\begin{gathered}
\kappa=\left[\begin{array}{lllllll}
f_{0,0,1} & f_{0,1,0} & f_{0,1,1} & f_{1,0,0} & f_{1,0,1} & f_{1,1,0} & f_{1,1,1}
\end{array}\right]^{T}, \\
\Phi=\left[\begin{array}{lllllll}
f_{0,0,2} & f_{0,1,1} & f_{0,1,2} & f_{1,0,1} & f_{1,0,2} & f_{1,1,1} & f_{1,1,2} \\
f_{0,1,1} & f_{0,2,0} & f_{0,2,1} & f_{1,1,0} & f_{1,1,1} & f_{1,2,0} & f_{1,2,1} \\
f_{0,1,2} & f_{0,2,1} & f_{0,2,2} & f_{1,1,1} & f_{1,1,2} & f_{1,2,1} & f_{1,2,2} \\
f_{1,0,1} & f_{1,1,0} & f_{1,1,1} & f_{2,0,0} & f_{2,0,1} & f_{2,1,0} & f_{2,1,1} \\
f_{1,0,2} & f_{1,1,1} & f_{1,1,2} & f_{2,0,1} & f_{2,0,2} & f_{2,1,1} & f_{2,1,2} \\
f_{1,1,1} & f_{1,2,0} & f_{1,2,1} & f_{2,1,0} & f_{2,1,1} & f_{2,2,0} & f_{2,2,1} \\
f_{1,1,2} & f_{1,2,1} & f_{1,2,2} & f_{2,1,1} & f_{2,1,2} & f_{2,2,1} & f_{2,2,2}
\end{array}\right]
\end{gathered}
$$

in which

$$
f_{s, p, q}=\sum_{n=0}^{N-1} n^{s} \sum_{k=0}^{K-1}\left(d_{k}^{(T)}\right)^{p} \sum_{l=0}^{L-1}\left(d_{l}^{(R)}\right)^{q}
$$

Plugging the above results into (20) yields

$$
\operatorname{SNR}_{K}=\frac{\lambda_{K}\left(\left|\alpha_{1}\right|^{2}+\left|\alpha_{2}\right|^{2}\right)}{2 \zeta^{H}\left(\Phi-N /(K L) \kappa \kappa^{H}\right) \zeta} .
$$

3.2. Case of Unknown Noise Variance. Next, we extend the analysis in the previous subsection to the case of unknown noise variance, under which the GLRT has the following form [19]:

$$
G(\mathbf{y})=\frac{p\left(\mathbf{y} ; \widehat{\boldsymbol{\alpha}}_{H_{1}}, \widehat{\boldsymbol{\zeta}}, \widehat{\sigma}_{H_{1}}^{2}, H_{1}\right)}{p\left(\mathbf{y} ; \widehat{\boldsymbol{\alpha}}_{H_{0}}, \widehat{\sigma}_{H_{0}}^{2}, H_{0}\right)}=\left(\frac{\left\|\widehat{\mathbf{z}}_{H_{0}}\right\|^{2}}{\left\|\widehat{\mathbf{z}}_{H_{1}}\right\|^{2}}\right)^{K L N},
$$

where $\widehat{\mathbf{z}}_{H_{0}}$ and $\widehat{\mathbf{z}}_{H_{1}}$ have the same definitions as that in (16). For the convenience of derivation, we define another statistic based on the GLRT as

$$
\begin{aligned}
V(\mathbf{y}) & =\ln \left(G(\mathbf{y})^{1 / K L N}\right)-1 \\
& =\frac{2 \mathbf{y}^{H}\left(P_{\mathbf{D}}^{\perp}-P_{[\mathbf{G D}]}^{\perp}\right) \mathbf{y} / \sigma^{2}}{2 \mathbf{y}^{H} P_{[\mathbf{G D}]}^{\perp} \mathbf{y} / \sigma^{2}} \triangleq \frac{T_{K}(\mathbf{y})}{N(\mathbf{y})} .
\end{aligned}
$$

Similarly as before, the projector $P_{[\mathbf{G D}]}^{\perp}$ is decomposed as $P_{[\mathbf{G D}]}^{\perp}=\mathbf{U}^{\prime} \mathbf{U}^{\prime H}$, where $\mathbf{U}^{\prime}$ is an unitary matrix. Hence $N(\mathbf{y})=$ $2 \mathbf{y}^{H} \mathbf{U}^{\prime} \mathbf{U}^{\prime H} \mathbf{y} / \sigma^{2} \triangleq 2\|\widehat{\mathbf{y}}\|^{2} / \sigma^{2}$, which in turn implies

$$
N(\mathbf{y}) \sim\left\{\begin{array}{l}
H_{0}: \chi_{2 r^{\prime}}^{2} \\
H_{1}: \chi_{2 r^{\prime}}^{2}
\end{array}\right.
$$

where $r^{\prime}=\operatorname{rank}\left(P_{[\mathbf{G D}]}^{\perp}\right)=K L N-M-6$. Recall that the statistical property of $T_{K}(\mathbf{y})$ has been given in (17); moreover, it is proved that $T_{K}(\mathbf{y})$ and $N(\mathbf{y})$ are independent under both hypotheses [9]; hence substituting (17) and (27) into (26) immediately suggests that

$$
T_{U}(\mathbf{y}) \triangleq \frac{T_{K}(\mathbf{y}) / r}{N(\mathbf{y}) / r^{\prime}} \sim\left\{\begin{array}{l}
H_{0}: F_{2 r, 2 r^{\prime}} \\
H_{1}: F_{2 r, 2 r^{\prime}}\left(\lambda_{U}\right)
\end{array}\right.
$$

where $F_{2 r, 2 r^{\prime}}$ and $F_{2 r, 2 r^{\prime}}\left(\lambda_{U}\right)$ denote the central and noncentral $F$ distributions [19], respectively, in which $2 r$ and $2 r^{\prime}$ are the DOFs of the $F$-distribution and the noncentrality parameter $\lambda_{U}$ has the same analytical expression as that in (18). Similarly, $\lambda_{U}$ can be numerically computed as the solution of

$$
Q_{F_{2 r, 2 r^{\prime}}^{-1}}\left(P_{F}\right)=Q_{F_{2 r, 2 r^{\prime}}\left(\lambda_{U}\right)}^{-1}\left(P_{D}\right)
$$

Consequently, the resolution threshold is

$$
\mathrm{SNR}_{U}=\frac{\lambda_{U}\left(\left|\alpha_{1}\right|^{2}+\left|\alpha_{2}\right|^{2}\right)}{2 \zeta^{H} \mathbf{G}^{H} P_{\mathbf{D}}^{\perp} \mathbf{G} \zeta} .
$$

As for the clutter free case, the expression of $\mathrm{SNR}_{U}$ can be obtained simply by substituting $\lambda_{K}$ in (24) with $\lambda_{U}$. 
3.3. The Ideal (Clairvoyant) Detector. The GLRT was adopted in previous subsections due to the fact that there exist unknown parameters in the observation model; that is, $\zeta$ and $\boldsymbol{\alpha}$ (possibly $\sigma^{2}$ ) in (14). Next we discuss the ideal situation that all these parameters are known as a prior. The result will be used as a performance evaluation indicator in the numerical examples.

Define another observation as $\mathbf{y}^{\prime}=\mathbf{y}-\mathbf{D} \boldsymbol{\alpha}$; thus we have

$$
\mathbf{y}^{\prime} \sim\left\{\begin{array}{l}
H_{0}: \mathscr{C} \mathscr{N}\left(\mathbf{0}, \sigma^{2} \mathbf{I}\right) \\
H_{1}: \mathscr{C} \mathscr{N}\left(\mathbf{G} \boldsymbol{\zeta}, \sigma^{2} \mathbf{I}\right) .
\end{array}\right.
$$

Therefore, the log-LRT statistic (omit the constant term) and its distribution are shown as [19]

$$
\begin{aligned}
T_{C}(\mathbf{y}) & \triangleq \operatorname{Re}\left(\mathbf{y}^{\prime H} \mathbf{G} \zeta\right) \\
& \sim\left\{\begin{array}{l}
H_{0}: \mathcal{N}\left(\mathbf{0}, \frac{\sigma^{2} \boldsymbol{\zeta}^{H} \mathbf{G}^{H} \mathbf{G} \zeta}{2}\right) \\
H_{1}: \mathcal{N}\left(\boldsymbol{\zeta}^{H} \mathbf{G}^{H} \mathbf{G} \boldsymbol{\zeta}, \frac{\sigma^{2} \zeta^{H} \mathbf{G}^{H} \mathbf{G} \zeta}{2}\right) .
\end{array}\right.
\end{aligned}
$$

Consequently, the resolution threshold is

$$
\mathrm{SNR}_{C}=\frac{\left(Q^{-1}\left(P_{F}\right)-Q^{-1}\left(P_{D}\right)\right)^{2}\left(\left|\alpha_{1}\right|^{2}+\left|\alpha_{2}\right|^{2}\right)}{2 \zeta^{H} \mathbf{G}^{H} \mathbf{G} \zeta} .
$$

\section{Numerical Examples}

In this section, the resolution thresholds derived in the previous section are analyzed through numerical examples combined with theoretical explanations. We first illustrate the necessity of the extension explored in this work through a somewhat extreme example. Subsequently, the effects of four factors on the resolution performance are investigated successively. Without loss of generality and for the clarity of illustration, we only consider the case of known noise variance except for that in Section 4.2 and clutter sources are involved only in Section 4.5. In addition, we make the following assumptions in the simulations.

(1) Both the transmit and the receive arrays are standard linear array (SLA) [16] with four elements; that is, $K=$ $L=4, d_{i}^{(T)}=d_{i}^{(R)}=i \lambda / 2, i=0,1, \ldots, 3$, where the wavelength $\lambda=0.3 \mathrm{~m}$.

(2) $\left(w_{c}^{(D)}, w_{c}^{(T)}, w_{c}^{(R)}\right)=(0,0,0)$, and $\left(P_{F}, P_{D}\right)=(0.001$, 0.999).

(3) The scattering coefficients of the targets satisfy $\left(\alpha_{1}\right.$, $\left.\alpha_{2}\right)=\left(1, e^{j \pi}\right)$ except for that in Section 4.4.

(4) The accumulated pulse number is $N=16$ except for that in Section 4.3.

4.1. Resolution Based on Doppler Frequency Difference. Consider an extreme case that the space positions of the two targets to be resolved are overlapped, which in turn implies that $\left(\delta_{T}, \delta_{R}\right)=(0,0)$. It is obvious that this is among the various cases that the scheme in [9] is incapable of. Figure 2

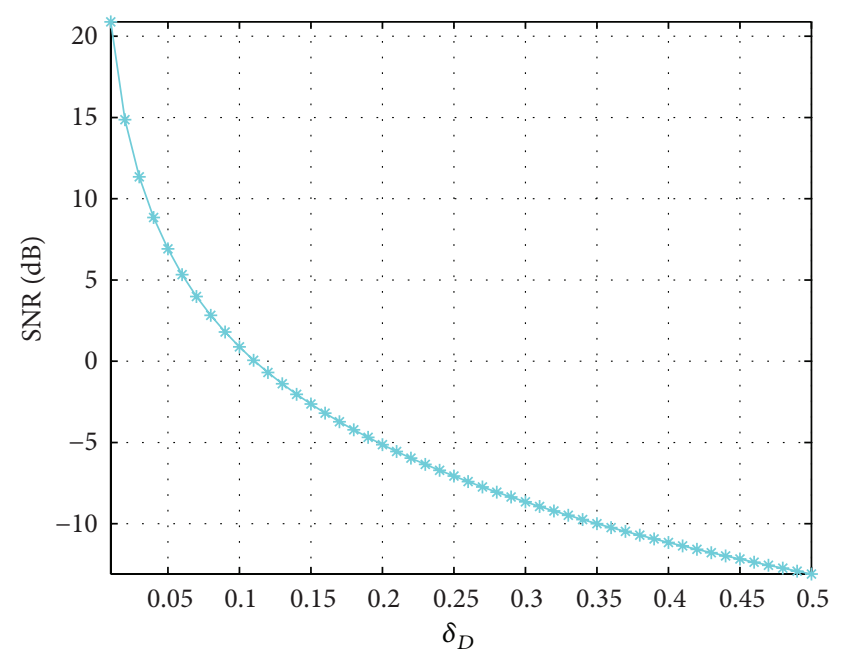

FIGURE 2: The SRL in the Doppler dimension versus the resolution threshold.

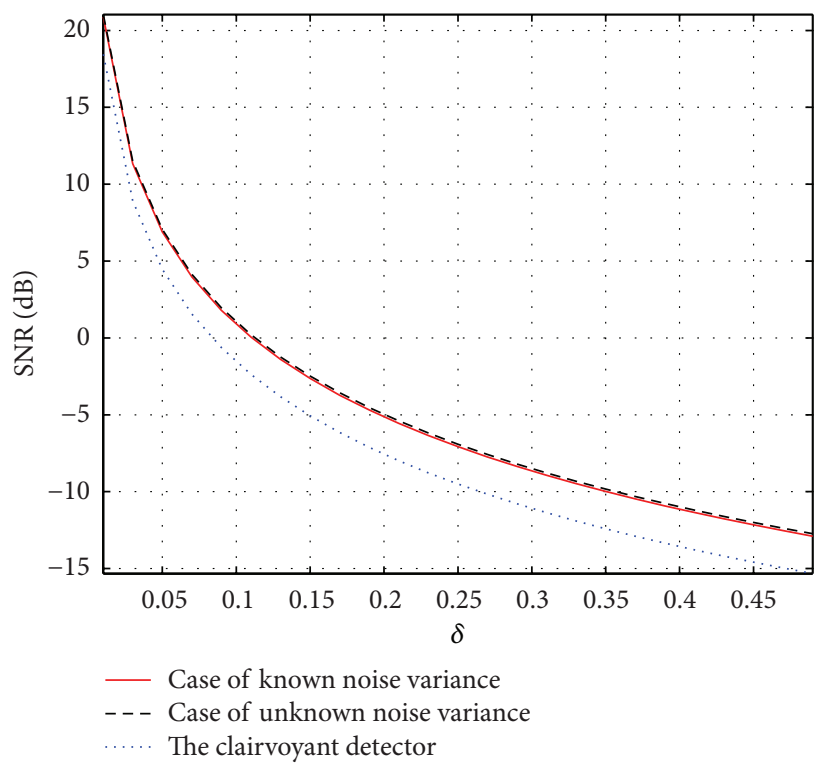

FIgURE 3: The SRL versus the resolution threshold.

reveals that the Doppler information can indeed be exploited to accomplish the resolution and as the difference between the two targets' Doppler frequencies increases, the difficulty on discriminating them decreases rapidly.

4.2. Effect of the Parameters' Prior. In this subsection we assume that the SRLs in each dimension are equal, that is, $\delta_{T}=\delta_{R}=\delta_{D} \triangleq \delta$, based on which we compare the resolution threshold in (1) the case of known noise variance, (2) the case of unknown noise variance, and (3) the clairvoyant detector, respectively.

As is shown in Figure 3, $\mathrm{SNR}_{C}<\mathrm{SNR}_{K}<\mathrm{SNR}_{U}$ holds, which is also consistent with intuition. Particularly, two facts are worth mentioning. Firstly, the difference between the first two cases is small; actually, the gap between the two curves is 


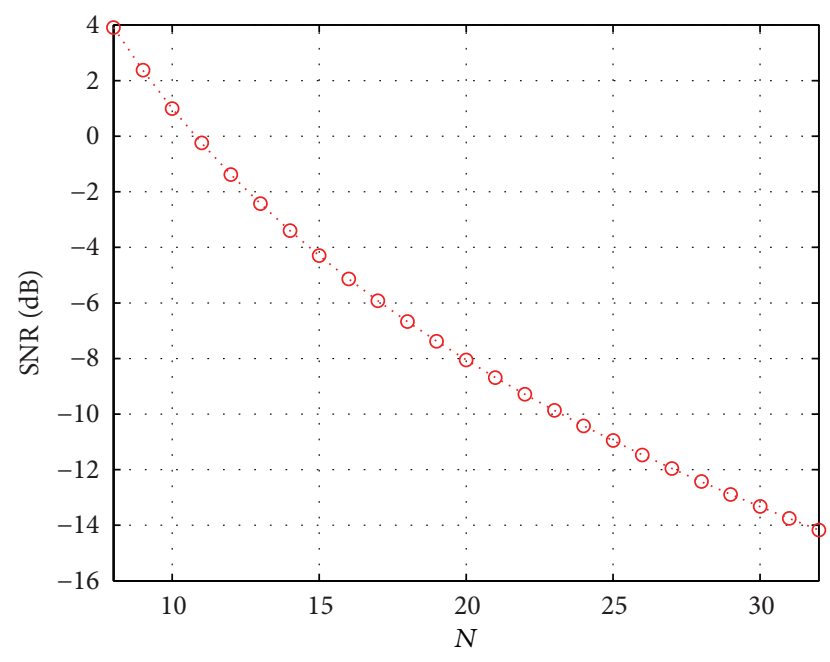

FIGURE 4: The accumulated pulse number versus the resolution threshold.

evaluated at about $0.16 \mathrm{~dB}$. Besides, the asymptotic behavior of the three curves is almost the same; in other words, the priors on the parameters become insignificant in the high SNR region.

4.3. Effect of the Accumulated Pulse Number. Next we examine the influence of the accumulated pulse number on the resolution threshold. The SRLs in each dimension are fixed in this subsection; that is, $\delta_{T}=\delta_{R}=\delta_{D}=0.2$. As illustrated in Figure 4, the resolution threshold decreases monotonically with the pulse number increases. More precisely, when $N$ ranges from 8 to 32 , the resolution threshold decreases by $17 \mathrm{~dB}$. At first glance, the result seems intuitional since, in conventional radar context, the accumulation of pulse means the improvement of the SNR. But note that the so-called coherent accumulation gain [23] is only $10 \log 10(32 / 8)=$ $6 \mathrm{~dB}$ in this case. In other words, from the resolution point of view, the SNR gain will be more efficient when accumulating pulses. An intuitive interpretation is that the increase of the pulse number means (1) accumulation of signal energy and (2) enhancement of the intrinsic Doppler resolution. These two factors combined lead to this phenomenon.

4.4. Effect of the Correlation between the Scattering Coefficients of the Two Targets. In this subsection, we assume that the total power of the echoes from the two targets is fixed, which translates into $\left|\alpha_{1}\right|^{2}+\left|\alpha_{2}\right|^{2}=C$, where $C$ is a constant. Without loss of generality, we set $C=1$ in the simulation. Similarly as in the previous subsection, $\delta_{T}=\delta_{R}=\delta_{D}=0.2$ holds. We focus on how the relative intensity and the phase difference of the two scattering coefficients affect the resolution threshold and the results are shown in Figure 5. Particularly, Figure 5(a) reveals that when the two targets are equipowered, that is, $\left|\alpha_{1}\right|^{2}=\left|\alpha_{2}\right|^{2}=0.5$, the resolution threshold reaches the peak, which also means that the two targets are most difficult to resolve. In addition, Figure 5(b) indicates that when the two

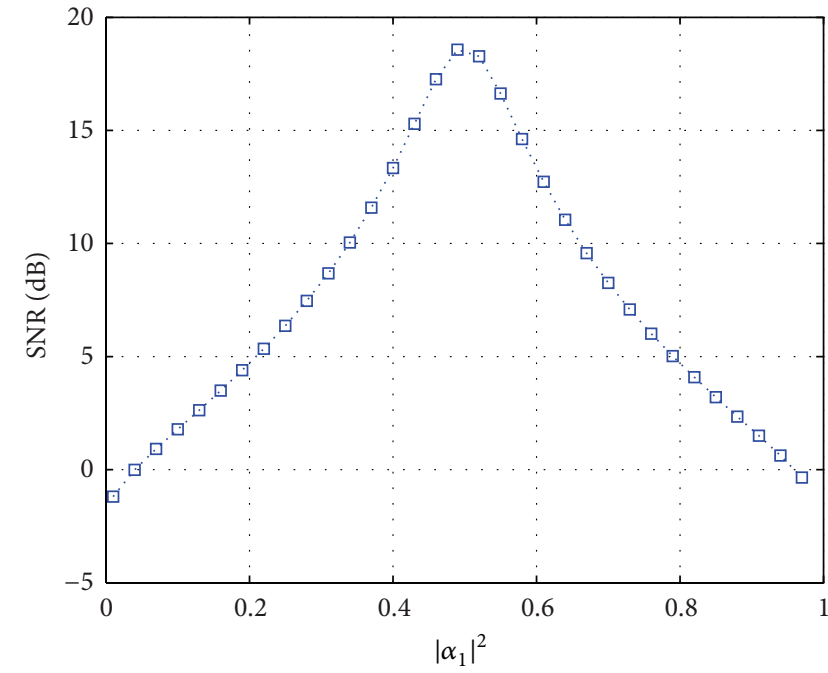

(a)

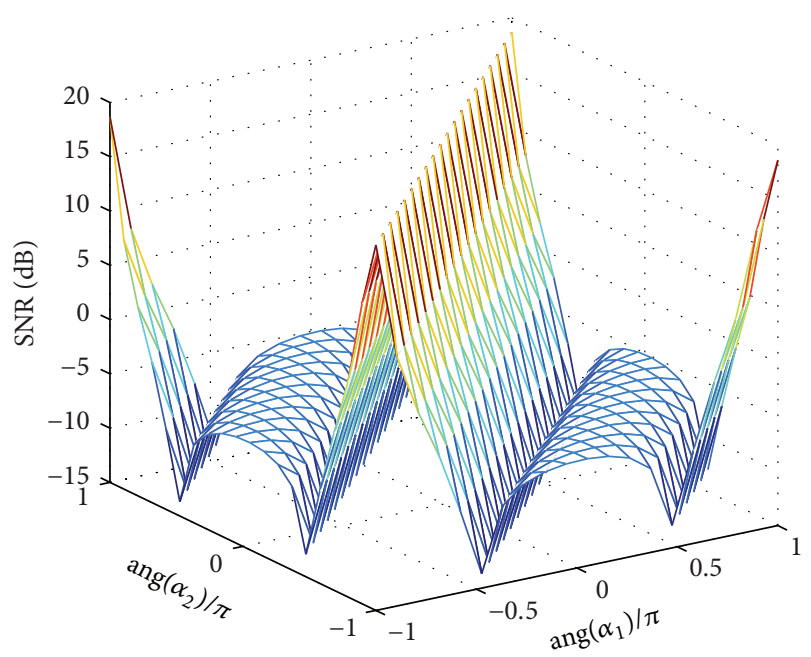

(b)

FIGURE 5: (a) The intensity of the scattering coefficient of the first target versus the resolution threshold $\left(\operatorname{ang}\left(\alpha_{1}\right)=\operatorname{ang}\left(\alpha_{2}\right)=0\right)$. (b) The phases of the two scattering coefficients versus the resolution threshold $\left(\left|\alpha_{1}\right|^{2}=\left|\alpha_{2}\right|^{2}=0.5\right)$.

scattering coefficients are inphase, the highest SNR is needed to resolve the two targets.

4.5. Effect of the Clutter Sources. The resolution performance may be deteriorated by the existence of clutter sources, which is reflected in the rise of the resolution threshold or equivalently the SNR loss. For sake of brevity, only two clutters with their scattering coefficients being 1 are introduced, namely, $M=4$ and $\left(\alpha_{3}, \alpha_{4}\right)=(1,1)$. Without loss of generality and for the clarity of illustration, only the effects of the clutters' Doppler frequencies are studied. Besides, we set $\delta_{T}=\delta_{R}=$ $\delta_{D}=0.2, w_{3}^{(T)}=w_{4}^{(T)}=w_{c}^{(T)}$, and $w_{3}^{(R)}=w_{4}^{(R)}=w_{c}^{(R)}$ in the simulation. The SNR loss is plotted as a function of the Doppler frequencies of the two clutter sources in Figure 6 and a significant feature of which is the three grooves indicated 


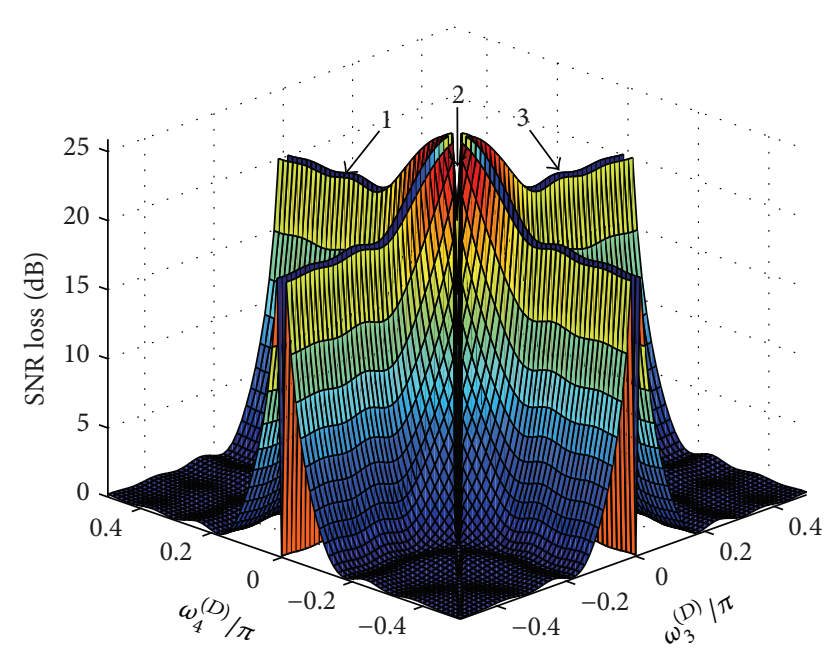

FIGURE 6: The Doppler frequencies of the two clutter sources versus the SNR loss.
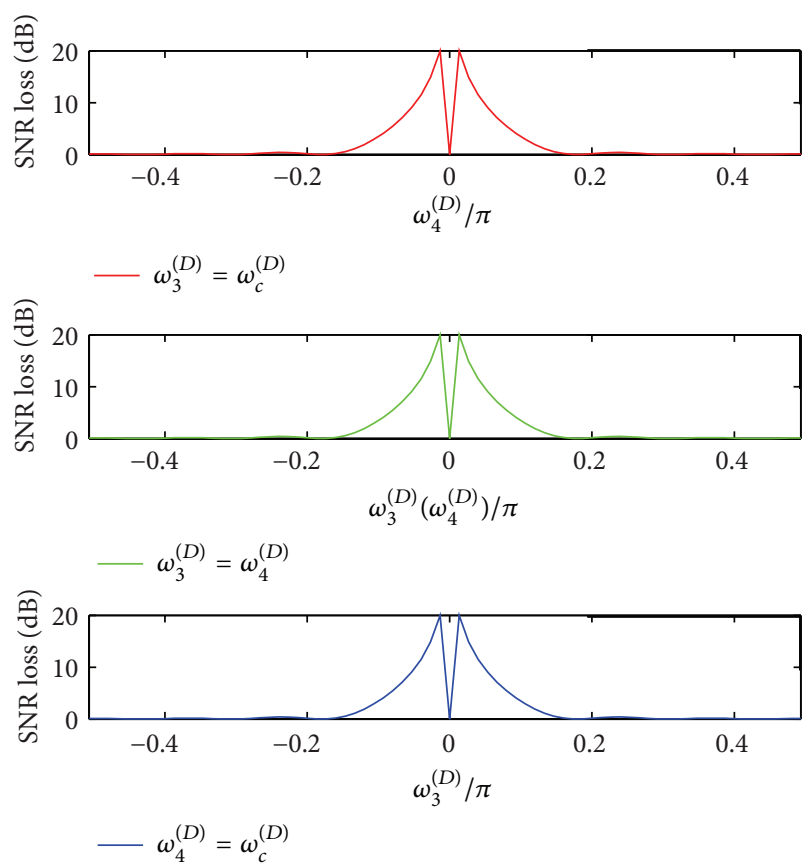

FIGURE 7: Sectional drawings of the three grooves in Figure 6.

by the arrows. The sectional drawings of them are shown in Figure 7 and they correspond to the following three cases:

(1) $w_{3}^{(D)}=w_{c}^{(D)}$;

(2) $w_{3}^{(D)}=w_{4}^{(D)}$;

(3) $w_{4}^{(D)}=w_{c}^{(D)}$.

An intuitive interpretation for such phenomenon can be given as follows: $\mathbf{g}_{3}$ or $\mathbf{g}_{4}$ equals $\mathbf{v}_{1}$ for case (1) and (3). As described in Section 3.1, an immediate consequence is that the first or the second clutter appears to be transparent, thus mitigating the SNR loss. As for case (2), the two clutters are overlapped and merge into a single one with greater intensity. Recall that through investigating the analytical expression of the resolution threshold, we have revealed that the intensities of the clutters have no influence on the resolution performance, apparently that the effect will be weakened in this situation. In sum, the simulation results well coincide with the theoretical analyses. Besides, another significant trend obtained from Figure 6 is that, with the Doppler frequencies of the clutters deviating from $w_{c}^{(D)}$, the influence recedes, which is also consistent with intuition. We would emphasize that the aforementioned conclusions are done for $w^{(T)}$ and $w^{(R)}$ and the results are omitted here due to space limit.

\section{Conclusion}

In this paper, the resolution performance of MIMO radar in terms of the 3D SRL is investigated where both the DOA and DOD combined with the Doppler frequency are employed for resolving two point targets of interest. Based on a GLRT formulation, the closed-form expressions of the resolution thresholds in a broadband noise background with/without clutter sources are derived. Both theoretical and numerical analyses are presented to provide an in-depth understanding of the resolution limit. Particularly, we have the following three interesting conclusions.

(1) From the resolution point of view, the profit in terms of SNR obtained by accumulating pulses is far better than the conventional coherent accumulation gain.

(2) Suppose that the total power of the echoes from the two targets of interest is fixed; then the worst situation for them to be resolved is $\alpha_{1}=\alpha_{2}$; namely, they share the same scattering coefficient.

(3) The influences of the clutters on the resolution performance are fully embodied in the structure of the remaining clutter space defined in Section 3.1.

\section{Conflict of Interests}

The authors declare that there is no conflict of interests regarding the publication of this paper.

\section{References}

[1] J. Li and P. Stoica, "MIMO radar with colocated antennas," IEEE Signal Processing Magazine, vol. 24, no. 5, pp. 106-114, 2007.

[2] A. M. Haimovich, R. S. Blum, and L. J. Cimini, "MIMO radar with widely separated antennas," IEEE Signal Processing Magazine, vol. 25, no. 1, pp. 116-129, 2008.

[3] I. Bekkerman and J. Tabrikian, "Target detection and localization using MIMO radars and sonars," IEEE Transactions on Signal Processing, vol. 54, no. 10, pp. 3873-3883, 2006.

[4] B. Tang, J. Tang, and Y. Peng, "MIMO radar waveform design in colored noise based on information theory," IEEE Transactions on Signal Processing, vol. 58, no. 9, pp. 4684-4697, 2010.

[5] H. Godrich, A. M. Haimovich, and R. S. Blum, "Target localization accuracy gain in MIMO radar based systems," IEEE Transactions on Information Theory, vol. 56, no. 6, pp. 27832803, 2010 . 
[6] H. Godrich, V. M. Chiriac, A. M. Haimovich, and R. S. Blum, "Target tracking in MIMO radar systems: Techniques and performance analysis," in Proceedings of the IEEE International Radar Conference (RADAR '10), pp. 1111-1116, Washington, DC, USA, May 2010.

[7] N. H. Lehmann, A. M. Haimovich, R. S. Blum, and L. Cimini, "High resolution capabilities of MIMO radar," in Proceedings of the 40th Asilomar Conference on Signals, Systems, and Computers (ACSSC '06), pp. 25-30, November 2006.

[8] M. N. E. Korso, R. Boyer, A. Renaux, and S. Marcos, "Statistical resolution limit for the multidimensional harmonic retrieval model: hypothesis test and cramer-rao bound approaches," EURASIP Journal on Advances in Signal Processing, vol. 2011, article 12, pp. 1-14, 2011.

[9] M. N. El Korso, R. Boyer, A. Renaux, and S. Marcos, "Statistical resolution limit for source localization with clutter interference in a MIMO radar context," IEEE Transactions on Signal Processing, vol. 60, no. 2, pp. 987-992, 2012.

[10] M. Shahram and P. Milanfar, "Imaging below the diffraction limit: a statistical analysis," IEEE Transactions on Image Processing, vol. 13, no. 5, pp. 677-689, 2004.

[11] M. Shahram and P. Milanfar, "On the resolvability of sinusoids with nearby frequencies in the presence of noise," IEEE Transactions on Signal Processing, vol. 53, no. 7, pp. 2579-2588, 2005.

[12] E. Dilaveroglu, "Nonmatrix cramér-rao bound expressions for high-resolution frequency estimators," IEEE Transactions on Signal Processing, vol. 46, no. 2, pp. 463-474, 1998.

[13] S. T. Smith, "Statistical resolution limits and the complexified Cramér-Rao bound," IEEE Transactions on Signal Processing, vol. 53, no. 5, pp. 1597-1609, 2005.

[14] M. N. E. Korso, R. Boyer, A. Renaux, and S. Marcos, "Deterministic performance bounds on the mean square error for near field source localization," IEEE Transactions on Signal Processing, vol. 61, no. 4, pp. 871-877, 2013.

[15] K. Sharman and T. Durrani, "Resolving power of signal subspace methods for fnite data lengths," in Proceedings of the IEEE International Conference on Acoustics, Speech, and Signal Processing, Detroit, Mich, USA, 1995.

[16] H. L. van Trees, Optimum Array Processing: Part IV of Detection, Estimation, and Modulation Theory, John Wiley \& Sons, New York, NY, USA, 2002.

[17] K. Sharman and T. Durrani, "Resolving power of signal subspace methods for fnite data lengths," in Proceedings of the IEEE International Conference on Acoustics, Speech, and Signal Processing, pp. 1501-1504, Tampa, Fla, USA, 1985.

[18] M. N. El Korso, R. Boyer, A. Renaux, and S. Marcos, "Statistical resolution limit for multiple parameters of interest and for multiple signals," in Proceedings of the IEEE International Conference on Acoustics, Speech, and Signal Processing (ICASSP '10), pp. 3602-3605, Dallas, Tex, USA, March 2010.

[19] S. M. Kay, Fundamentals of Statistical Signal Processing: Detection Theory, vol. 2, Prentice Hall, Upper Saddle River, NJ, USA, 1998.

[20] Z. Liu and A. Nehorai, "Statistical angular resolution limit for point sources," IEEE Transactions on Signal Processing, vol. 55, no. 11, pp. 5521-5527, 2007.

[21] H. B. Lee and M. S. Wengrovitz, "Resolution threshold of beamspace MUSIC for two closely spaced emitters," IEEE Transactions on Acoustics, Speech, and Signal Processing, vol. 38, no. 9, pp. 1545-1559, 1990.
[22] R. Boyer, "Performance bounds and angular resolution limit for the moving colocated MIMO radar," IEEE Transactions on Signal Processing, vol. 59, no. 4, pp. 1539-1552, 2011.

[23] X. W. Tang, J. Tang, Q. He et al., "Cramer-Rao Bounds and coherence performance analysis for next generation radar with pulse trains," Sensors, vol. 13, no. 4, pp. 5347-5367, 2013.

[24] M. N. E. Korso, R. Boyer, A. Renaux, and S. Marcos, "On the asymptotic resolvability of two point sources in known subspace interference using a GLRT-based framework," Signal Processing, vol. 92, no. 10, pp. 2471-2483, 2012.

[25] R. T. Behrens and L. L. Scharf, "Signal processing applications of oblique projection operators," IEEE Transactions on Signal Processing, vol. 42, no. 6, pp. 1413-1424, 1994. 

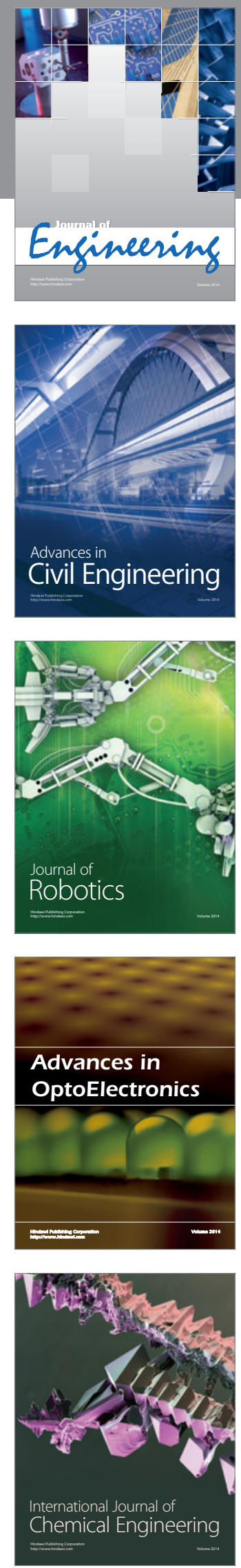

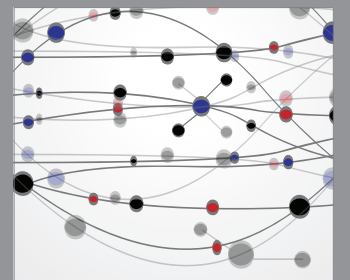

The Scientific World Journal
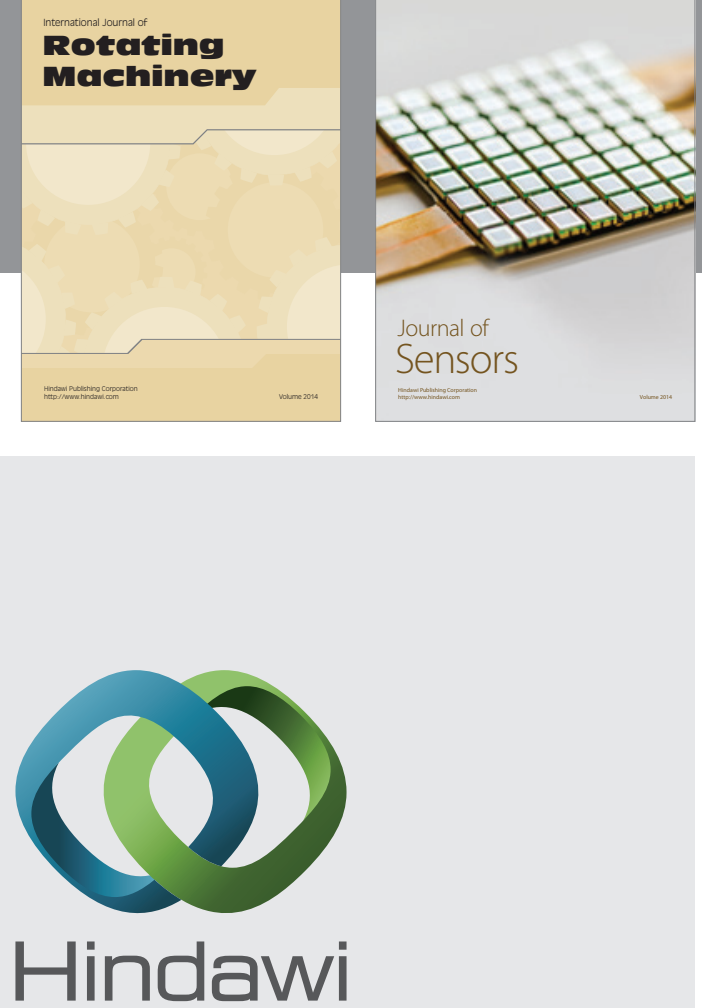

Submit your manuscripts at http://www.hindawi.com
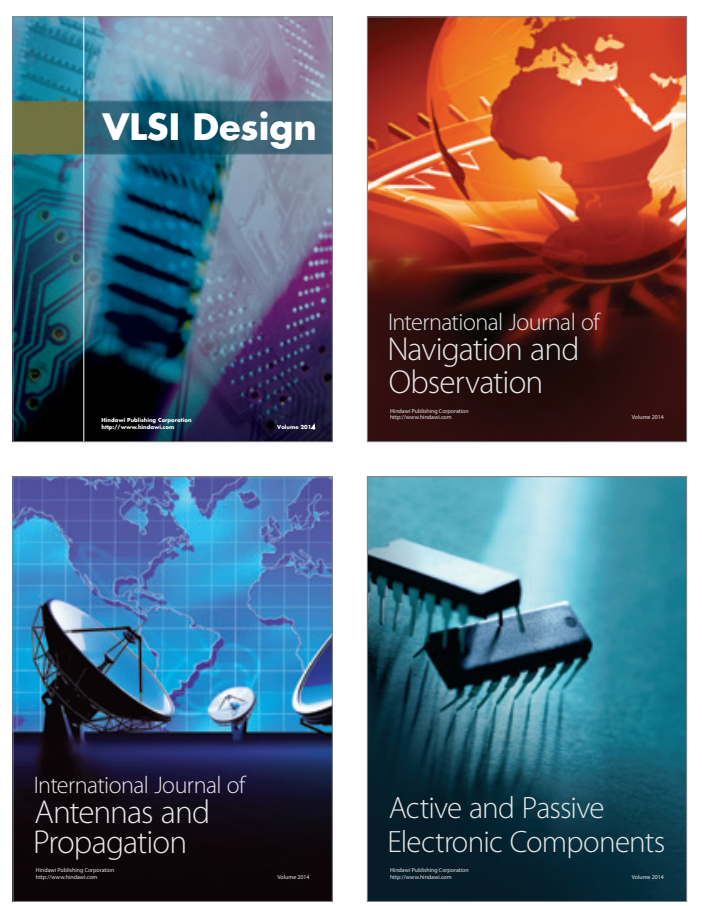
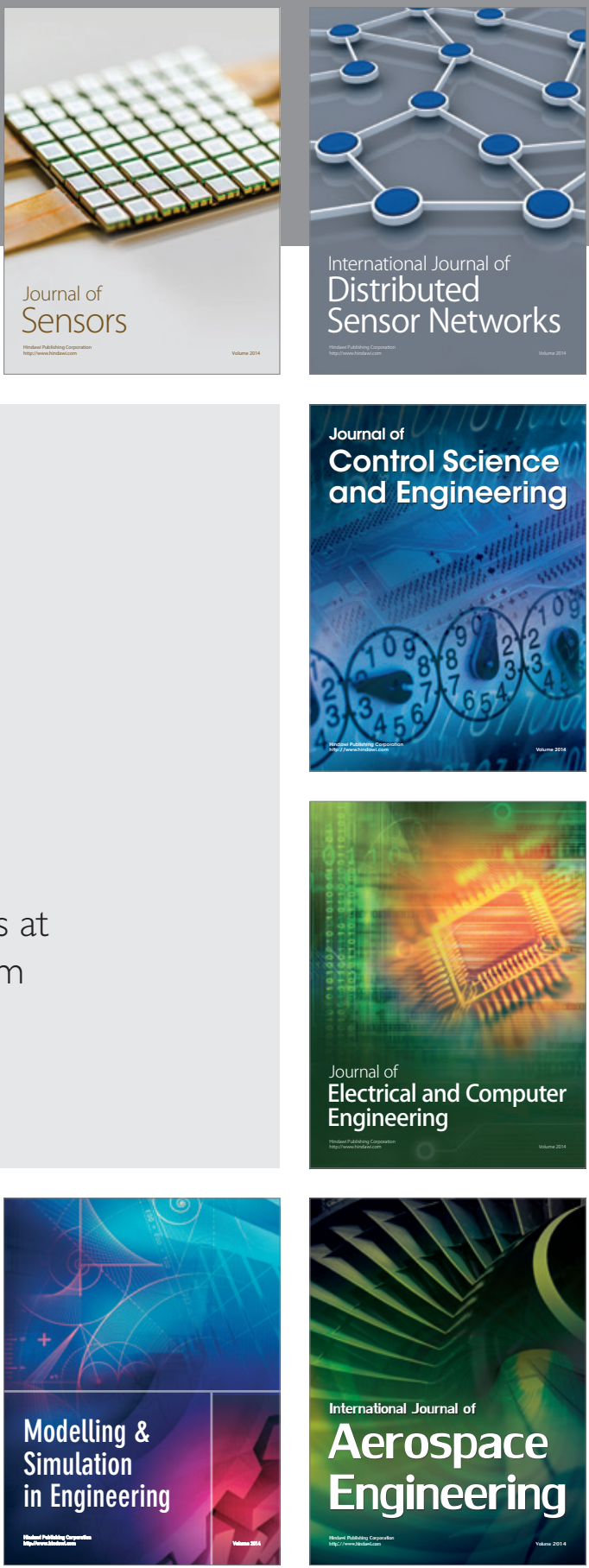

Journal of

Control Science

and Engineering
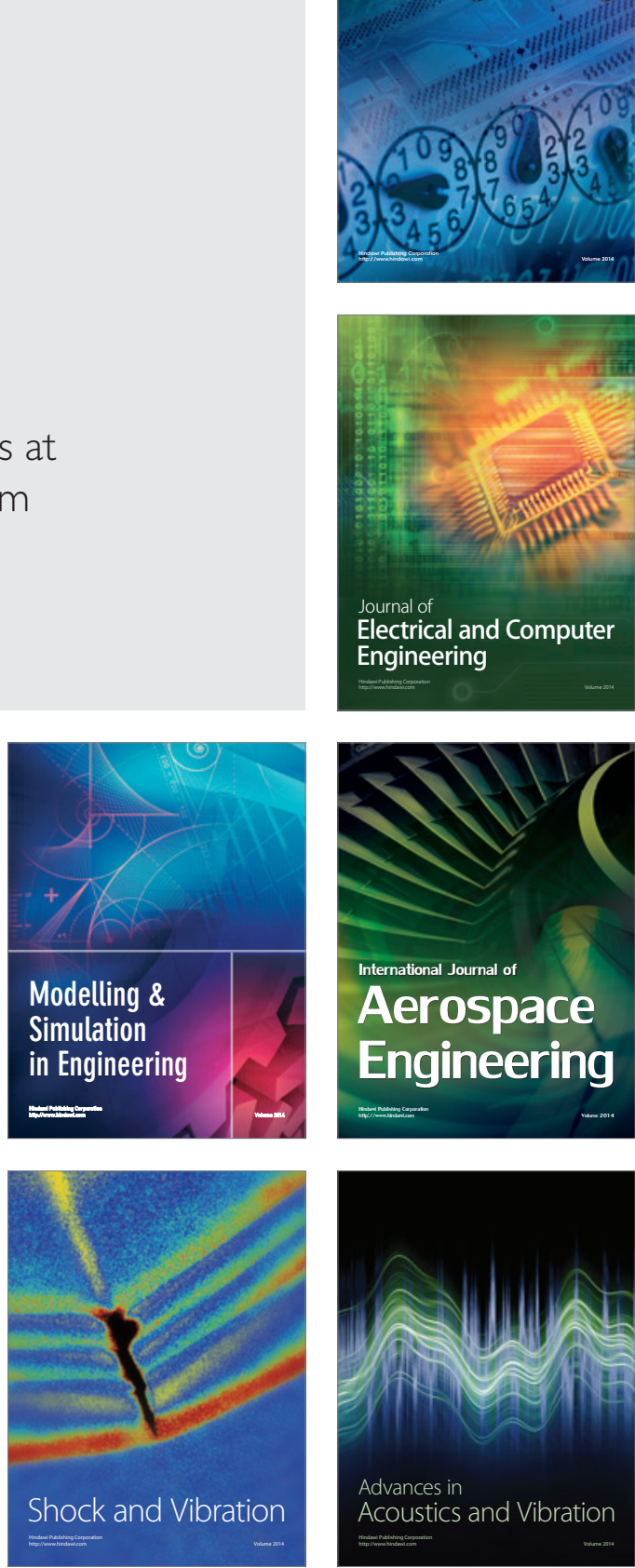